\title{
Pregnancy-related anxiety and depression during the coronavirus 2019 pandemic
}

\author{
Farzin Bagheri Sheykhangafshe* ${ }^{(\mathbb{D}}$ \\ PhD Candidate in Psychology, Faculty of Humanities, Tarbiat Modares University, Tehran, Iran
}

*Corresponding Author: Farzin Bagheri Sheykhangafshe, Department of Psychology, Faculty of Humanities, Tarbiat Modares University, Tehran, Iran. Email: farzinbagheri@modares.ac.ir

Received: 9 February 2021, Accepted: 24 February 2021, ePublished: 30 March 2021

\section{Dear Editor,}

Pregnancy is one of the most important events in women's life. It usually increases women's vulnerability to physical, psychological, and social problems, their physical and emotional needs, and the risk of anxiety and depression (1). During the first trimester of pregnancy, women experience problems such as fatigue, nausea, vomiting, and mood instability due to increased estrogen level. In the second trimester, nausea and vomiting usually disappear and women feel more comfortable, regain their previous strength, and feel excited due to the movements of the fetus. However, concerns about delivery, its outcomes, and fetal health in the third trimester cause them high levels of anxiety (2).

Besides pregnancy-related factors, there are many different factors which can affect pregnant women's mental health. These factors include epidemics, catastrophes, and disasters (3). The coronavirus disease 2019 (COVID-19) pandemic in recent years caused many different psychological problems for pregnant women. For example, during the severe acute respiratory syndrome coronavirus (SARS-CoV) outbreak in 2003, studies reported that pregnant women felt they were at higher risk for the disease, took strict preventive measures, and experienced high levels of anxiety (4). Similarly, pregnancy during the Middle East respiratory syndrome coronavirus (MERS-CoV) outbreak in 2012 was associated with many different physical and psychological problems and negative consequences for both pregnant women and their fetuses (1). During the Zika virus outbreak, many women were also worried and anxious about affliction by the disease and hence, experienced problems such as obsession and depression (5).

As the most recent pandemic, COVID-19 was first identified in Wuhan, China, in December 2019, and was introduced by the World Health Organization as a pandemic in March 11, 2020 (6). The symptoms of the COVID-19 include cold-like symptoms such as cough and dry throat. Problems associated with the COVID-19 pandemic such as multiple treatment failures, rumors, misinformation about disease origin, and frequent mutations of the virus have posed serious threat to public health (7) and caused fear, insecurity, stress, depression, and anxiety throughout the world, particularly among pregnant women. Moreover, suspension of occupational activities and enforcement of strict quarantine to reduce the spread of COVID-19 significantly changed personal and social activities and relations. Increasing number of deaths and the necessity of home quarantine during the pandemic were also associated with increasing prevalence of anxiety and stress for pregnant women (8).

The COVID-19 pandemic and its associated anxiety and stress may change pregnant women's healthcarerelated behaviors, negatively affect the health of their fetuses, and result in miscarriage or premature birth (9). A study into pregnancy-related anxiety during the COVID-19 pandemic revealed that $25.8 \%$ of women stopped face-to-face medical visits, $31.8 \%$ of them used video calls for receiving prenatal care, $12.3 \%$ of them did not want to give birth in hospital settings, $59.2 \%$ of them were worried about food shortage, $63.7 \%$ of them were worried about employment loss, and $93 \%$ of them reported increased rate of family conflicts. These problems had increased their anxiety and stress (3). Another study during the COVID-19 pandemic on 308 pregnant women with a mean age of 31 years also reported that $97.4 \%$ of them used face mask, $88.3 \%$ of them regularly washed their hands, and $76.3 \%$ of them were in home quarantine. The mean scores of their anxiety, social support, and risk perception were respectively $42.45,44.60$, and 21.60 , and those with adequate social support reported lower levels of anxiety during pregnancy (10). A study on 544 pregnant women and 315 non-pregnant women in China during the COVID-19 pandemic also showed that the prevalence rate of mental disorders among pregnant women was $5.3 \%$ for depression, $6.8 \%$ for anxiety, $2.4 \%$ for physical

(C) 2021 The Author(s); Published by Shahrekord University of Medical Sciences. This is an open-access article distributed under the terms of the Creative Commons Attribution License (https://creativecommons.org/licenses/by/4.0), which permits unrestricted use, distribution, and reproduction in any medium, provided the original work is properly cited. 
problems, $2.6 \%$ for insomnia, and $0.9 \%$ for posttraumatic stress, while non-pregnant women had lower levels of anxiety and depression (11). Moreover, a study during the COVID-19 pandemic on 1901 Chinese pregnant women with a mean age of 28 years showed that $34 \%$ of them had postpartum depression, $40 \%$ of them had posttraumatic stress, $96 \%$ of them were anxious about COVID-19, and $80 \%$ of them took preventive measures such as wearing face mask, frequent hand washing, and social distancing (2). Similarly, another study on pregnant women in China reported the increased prevalence of anxiety and depression after the COVID-19 pandemic and showed that women with greater anxiety about affliction by COVID-19 experienced higher postpartum depression (8).

The high prevalence of anxiety and depression during the COVID-19 pandemic highlights the necessity of taking appropriate measures to reduce mental problems $(6,7)$, particularly among pregnant women. Relaxation techniques can help prevent and reduce mental problems among pregnant women during the COVID-19 pandemic. Relaxation has positive effects on maternal and fetal health, reduces stress, depression, and anxiety, and provides deep feelings of physical and mental relaxation. It consists of diaphragmatic breathing and muscle relaxation exercises which reduce anxiety through influencing the hypothalamic-pituitary-adrenal axis (12). Given the unknown end of the COVID-19 pandemic, healthcare authorities and staff need to develop and use effective strategies to promote mental health among pregnant women.

\section{Conflict of Interests}

The authors declare no conflict of interests.

\section{Ethical Approval}

Not applicable.

\section{References}

1. Qiao Y, Wang J, Li J, Wang J. Effects of depressive and anxiety symptoms during pregnancy on pregnant, obstetric and neonatal outcomes: a follow-up study. J Obstet Gynaecol. 2012;32(3):237-40. doi: 10.3109/01443615.2011.647736.

2. Zhang CJP, Wu H, He Z, Chan NK, Huang J, Wang $\mathrm{H}$, et al. Psychobehavioral responses, post-traumatic stress and depression in pregnancy during the early phase of COVID-19 outbreak. Psychiatr Res Clin Pract. 2021;3(1):46-54. doi: 10.1176/appi.prcp.20200019.

3. Moyer CA, Compton SD, Kaselitz E, Muzik M.
Pregnancy-related anxiety during COVID-19: a nationwide survey of 2740 pregnant women. Arch Womens Ment Health. 2020;23(6):757-65. doi: 10.1007/s00737-020-01073-5.

4. Lee DT, Sahota D, Leung TN, Yip AS, Lee FF, Chung TK. Psychological responses of pregnant women to an infectious outbreak: a case-control study of the 2003 SARS outbreak in Hong Kong. J Psychosom Res. 2006;61(5):707-13. doi: 10.1016/j. jpsychores.2006.08.005.

5. Filgueiras Meireles JF, Neves CM, Morgado F, Caputo Ferreira ME. Zika virus and pregnant women: a psychological approach. Psychol Health. 2017;32(7):798-809. doi: 10.1080/08870446.2017.1307369.

6. Liang L, Gao T, Ren H, Cao R, Qin Z, Hu Y, et al. Post-traumatic stress disorder and psychological distress in Chinese youths following the COVID-19 emergency. J Health Psychol. 2020;25(9):1164-75. doi: $10.1177 / 1359105320937057$.

7. Amsalem D, Dixon LB, Neria Y. The coronavirus disease 2019 (COVID-19) outbreak and mental health: current risks and recommended actions. JAMA Psychiatry. 2021;78(1):9-10. doi: 10.1001/ jamapsychiatry.2020.1730.

8. Wu Y, Zhang C, Liu H, Duan C, Li C, Fan J, et al. Perinatal depressive and anxiety symptoms of pregnant women during the coronavirus disease 2019 outbreak in China. Am J Obstet Gynecol. 2020;223(2):240.e1240.e9. doi: 10.1016/j.ajog.2020.05.009.

9. Chen H, Guo J, Wang C, Luo F, Yu X, Zhang W, et al. Clinical characteristics and intrauterine vertical transmission potential of COVID-19 infection in nine pregnant women: a retrospective review of medical records. Lancet. 2020;395(10226):809-15. doi: 10.1016/s0140-6736(20)30360-3.

10. Yue C, Liu C, Wang J, Zhang M, Wu H, Li C, et al. Association between social support and anxiety among pregnant women in the third trimester during the coronavirus disease 2019 (COVID-19) epidemic in Qingdao, China: the mediating effect of risk perception. Int J Soc Psychiatry. 2021;67(2):120-7. doi: 10.1177/0020764020941567.

11. Zhou Y, Shi H, Liu Z, Peng S, Wang R, Qi L, et al. The prevalence of psychiatric symptoms of pregnant and non-pregnant women during the COVID-19 epidemic. Transl Psychiatry. 2020;10(1):319. doi: 10.1038/ s41398-020-01006-x.

12. Tragea C, Chrousos GP, Alexopoulos EC, Darviri C. A randomized controlled trial of the effects of a stress management programme during pregnancy. Complement Ther Med. 2014;22(2):203-11. doi: 10.1016/j.ctim.201 